\title{
The Product of the $\mathrm{t}(11 ; 18)$, an API2-MLT Fusion, Marks Nearly Half of Gastric MALT Type Lymphomas without Large Cell Proliferation
}

\author{
Mathijs Baens, ${ }^{*}$ Brigitte Maes, ${ }^{\dagger}$ Anja Steyls, ${ }^{\star}$ \\ Karel Geboes, ${ }^{\dagger}$ Peter Marynen, ${ }^{*}$ and \\ Christiane De Wolf-Peeters ${ }^{\dagger}$ \\ From the Human Genome Laboratory,* Center for Human \\ Genetics, Flanders Interuniversity Institute for Biotechnology, \\ Leuven; and the Department of Pathology, ${ }^{\dagger}$ University of Leuven, \\ Leuven, Belgium
}

Recently we demonstrated that the $t(11 ; 18)(q 21 ; q 21)$ associated with extranodal marginal zone $B$ cell lymphomas of MALT type results in the expression of a chimeric transcript fusing $5^{\prime} \boldsymbol{A P I} 2$ on chromosome 11 to $3^{\prime} M L T$ on chromosome 18 . Here we report the development of an RT-PCR approach for the detection of the API2-MLT fusion transcript and its application for the analysis of $\mathbf{5 8}$ cases of gastric lymphoma. Initially nested PCR amplification was combined with Southern analysis using internal API2 and MLT probes. A genuine $A P I 2-M L T$ fusion transcript of variable length was demonstrated in 11 out of 58 cases. Sequence analysis revealed that in all cases the breakpoint on chromosome 11 occurred between exons 7 and 8 of the API2 gene. In contrast, the breakpoints on chromosome 18 appeared to be heterogeneous as fusions to bp 814, 1123, and 1150, respectively, of MLT were observed. These observations allowed us to work out a highly sensitive diagnostic test for the API2-MLT fusion on an ABI Prism 7700 sequence detector that confirmed the results of our initial approach. The API2-MLT fusion was found in $48 \%$ of gastric marginal zone cell lymphomas of MALT type that did not contain a large cell component and it was lacking in all other lymphomas of the stomach. (Am J Patbol 2000, 156:1433-1439)

Mucosa-associated lymphoid tissue (MALT type) lymphoma has been recognized as a distinct entity within the marginal zone cell lymphomas listed in the REAL classification. ${ }^{1}$ This Iymphoma mimics the lymphoid follicles of Peyer's patches of the intestine and the B follicles of the white pulp of the spleen in both its growth pattern and its cellular composition. The tumor comprises B cell follicles each with a center and a lymphocytic corona composed of reactive $B$ cells and expanded marginal zones harboring the neoplastic population. MALT type lymphoma mainly affects extranodal sites among which the stomach is one of the most frequently involved organs. ${ }^{2}$ In this particular location its occurrence has been associated with Helicobacter pylori infection ${ }^{3}$ and with the occurrence of a large cell component. It has been postulated that the latter finding represents the progression from a low grade disease into a high grade malignancy indicated as "Iow grade" MALT type lymphoma with a "high grade" component or high grade MALT. ${ }^{4}$ This assumption has inspired others to speculate that all gastric lymphomas, diffuse large B cell lymphomas included, could be considered to be of MALT type. ${ }^{5}$ The latter hypothesis certainly demands support by firm experimental evidence. $^{6,7}$

Controversy also exists concerning the necessity to distinguish MALT type lymphoma from nodal and splenic marginal zone cell lymphoma. All three marginal zone cell lymphomas are considered to arise from a common normal counterpart, the marginal zone cell. Indeed they share similar morphological features and an identical immunophenotype. Moreover, and despite the frequent failure of cytogenetic analysis in this malignancy, a comparable cytogenetic profile of the three subtypes of marginal zone cell lymphoma has been demonstrated, with a particularly high incidence of trisomy 3 and trisomy 18.8,9 On the other hand, translocation $t(11 ; 18)$, one of the rarely found structural anomalies in marginal zone cell lymphomas, has been reported to occur exclusively in extranodal, low grade MALT type marginal zone cell lymphomas. ${ }^{10}$

Using two cases of gastric MALT type lymphoma documented by the $t(11 ; 18)(q 21 ; q 21)$ we recently succeeded in cloning the breakpoints on chromosome 11 and 18. Furthermore we demonstrated that the API2 gene, an inhibitor of apoptosis, and a novel gene on $18 q 21$, named $M L T$, are rearranged in this translocation. ${ }^{11}$ Based on these data a reverse transcription-poly-

Supported by a grant from the Belgian Cancer Association (to C. De W.-P.) and by grants G.0153.96 and G0377.97 from the Fonds voor Wetenschappelijk Onderzoek-Vlaanderen of Belgium (to P. M.). M. B. is a Postdoctoraal Onderzoeker and P. M. is an Onderzoeksdirecteur of the Fonds voor Wetenschappelijk Onderzoek-Vlaanderen, Belgium.

Accepted for publication January 6, 2000.

Address reprint requests to Prof. Christiane De Wolf-Peeters, Department of Pathology, University Hospitals, Catholic University of Leuven, Minderbroederstraat 12, B-3000 Leuven, Belgium. E-mail: Christiane. Peeters@uz.kuleuven.ac.be. 
Table 1. RT-PCR Primers

\begin{tabular}{|c|c|c|c|}
\hline \multicolumn{4}{|l|}{ Control PCR } \\
\hline GAPD247f & 5' AATCCCATCACCATCTTCCA & $\begin{array}{l}\text { GAPD596r } \\
\text { GAPD1147r }\end{array}$ & $\begin{array}{l}\text { 5' ACAGTCTTCTGGGTGGCAGT } \\
\text { 5' AGGGGAGATTCAGTGTGGTG }\end{array}$ \\
\hline A3f1 & 5' CAGCCCGCTTTAAAACATTC & MLT1464r & 5' ATGGATTTGGAGCATCAACG \\
\hline A3f2 & 5' AAACATTCTTTAACTGGCCCTCT & MLT1441r & 5' ACCATGAAGCTGTTCCCAAA \\
\hline A3ł3 & 5' TTGCAAGTGCGGGTTTTTAT & MLT1420r & 5' TTTTCATAACCATGTCCTGCAT \\
\hline \multicolumn{4}{|r|}{ 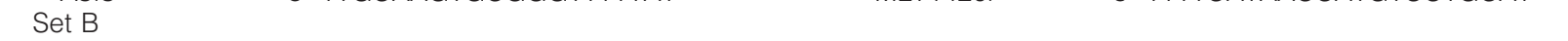 } \\
\hline A7f1 & 5' ATTAATGCTGCCGTGGAAAT & MLT2636r & 5' TTTTTCAGAAATTCTGAGCCTGTC \\
\hline A7f2 & 5' CCTGGTAAAACAGACAGTTCAGA & & \\
\hline \multicolumn{4}{|c|}{ Classic RT-PCR, MLT-API2 } \\
\hline \multicolumn{4}{|l|}{ Set C } \\
\hline A8r1 & 5' AACACAGCTTCAGCTTCTTGC & MLT374f & 5' TTGCCTAGACCTGGAGCAGT \\
\hline A8r2 & 5' TTAATAATTCCGGCAGTTAGTAGAC & MLT486f & 5' GATTTCCTGCAGGCTATGGA \\
\hline \multicolumn{4}{|c|}{ Real Time RT-PCR, API2-MLT } \\
\hline \multicolumn{4}{|l|}{ Set D } \\
\hline \multirow[t]{2}{*}{ A7f1 } & 5' ATTAATGCTGCCGTGGAAAT & MLT876r & 5' GCTTTTGGGAAGTTGGTTCA \\
\hline & MLT-845r & STTGGATTCA & ACGCCATCAACACT-TAMRA \\
\hline \multirow{2}{*}{$\begin{array}{l}\text { Set E } \\
\text { A7f1 }\end{array}$} & & & \\
\hline & MLT1197r & CCTTGTCCT & CAAAGGCTG-TAMRA \\
\hline
\end{tabular}

merase chain reaction (RT-PCR) approach was developed to detect $t(11 ; 18)(q 21 ; q 21)$ in a series of 58 consecutively received gastrectomy specimens demonstrating involvement by non-Hodgkin's lymphoma to define the incidence of this particular cytogenetic anomaly in gastric lymphomas and to clarify its role in the pathogenesis of MALT type lymphoma as well as in other lymphomas affecting the stomach.

\section{Materials and Methods}

\section{Patient Material}

All gastrectomy specimens involved by non-Hodgkin's lymphoma and documented by at least one representative freshly frozen tissue block were included in the study. These 58 consecutively received cases were collected in the department of pathology of the University Hospitals, Catholic University of Leuven over a period of 15 years. The series includes one case from a previous study. ${ }^{11}$ No cytogenetic data were available for the remaining cases.

In none of the cases was evidence of a lymphomatous process documented in the clinical history before the gastric complaints that led to the gastrectomy. Moreover, in most cases the neoplastic process was restricted to the stomach.

Histology was reviewed on paraffin-embedded, formalin-fixed material without knowledge of the RT-PCR findings. A minimum of two and a mean number of 10 blocks per specimen were available for the analysis. Diagnosis was based on hematoxylin and eosin (HE)-stained sections and on the results obtained using a panel of immunohistochemical stainings including pan-B cell and pan- $T$ cell staining, IgM, IgA, IgD, kappa, and lambda staining as well as CD5, CD10, and CD23 stainings performed on paraffin and/or frozen tissue sections. The lymphoma was subtyped according the International Lymphoma Study Group proposal or REAL classification. ${ }^{1}$ MALT type lym- phoma was diagnosed only in cases without clear evidence of a large cell proliferation. Gastrectomy specimens with sheets and clusters of large cells within the MALT type lymphoma, with large cells colonizing preexisting follicle centers or with a tumor mass composed of large cells only associated with MALT type lymphoma, were recorded as MALT type lymphoma and large cell proliferation. All cases composed exclusively of large cells were recorded as de novo diffuse large B cell lymphoma. Finally, the cases presenting as Burkitt's/ Burkitt's-like lymphoma are listed separately.

In 3 of the 58 cases, frozen mesenteric lymph node biopsies taken at the time of the gastrectomy and involved by the lymphoma were available for study.

\section{RT-PCR Analysis of the $t(11 ; 18)$ Fusion Transcripts}

Total RNA was extracted from no more than 10 tissue sections, $20 \mu \mathrm{m}$ thick, using Trizol reagent (Life Technologies, Mevelbeke, Belgium). These sections were taken from a representative frozen tissue block stored at $-80^{\circ} \mathrm{C}$ comprising the lymphomatous tissue as checked on an HE-stained frozen tissue section. Four hundred nanograms of total RNA was used as template for each RTPCR reaction (Titan one tube RT-PCR system, Boehringer Mannheim, Mannheim, Germany). Primers used in the different RT-PCR experiments are listed in Table 1.

The quality of the extracted RNA was verified by amplification of a 900-bp fragment of the GAPD gene using primers GAPD247f and GAPD1147r. For RT-PCR amplification of the API2-MLT fusion transcript, primer set A was used. In the first round of amplification, primer $A 3 f 1$, specific for exon 3 of API2, was used in combination with MLT1464r. A nested PCR was performed using primer A3f2 and MLT1441r. PCR products were size separated on $1.5 \%$ agarose gels and visualized by ethidium bro- 
Table 2. Frequency of the API2-MLT Fusion in Gastric Lymphomas

\begin{tabular}{lccc}
\hline \multicolumn{1}{c}{ Lymphoma type } & No. of cases & $\begin{array}{c}\text { API2-MLT fusion- } \\
\text { positive cases }\end{array}$ & $\%$ \\
\hline MALT & 23 & 11 & 48 \\
MALT with large cell component & 14 & 0 & 0 \\
Diffuse large cell lymphoma & 17 & 0 & 0 \\
Burkitt's/Burkitt's-like lymphoma & 4 & 0 & 0 \\
\hline
\end{tabular}

mide staining. Amplification products were blotted onto Hybond $\mathrm{N}+$ membranes with $0.4 \mathrm{~N} \mathrm{NaOH}$ and hybridized with ${ }^{32} \mathrm{P}$-labeled oligonucleotide probes A3f3, specific for exon 3 of $A P I 2$, and MLT1420r. Primer set $B$ was used to look for possible breakpoints further downstream in the $M L T$ gene. A heminested amplification was performed with primers $A 7 f 1$ and $A 7 f 2$ located in exon 7 of $A P I 2$ and $M L T 2636$, located at the stop codon of the gene. To evaluate the expression of the reciprocal MLT-API2 fusion, a nested RT-PCR/PCR (set C) was performed with primers $M L T 374 f$ and $M L T 486 f$ in combination with primers specific for exon 8 of API2, namely A8r 1 and A8r2.

PCR products were cloned in pGEM-T Easy (Promega, Madison, WI), nucleotide sequences were determined by dideoxy chain termination with fluorescein isothiocyanatelabeled primers and analyzed on an automated laser fluorescence sequencer (AP Biotech, Uppsala, Sweden).

\section{Real Time Detection of the API2-MLT Fusion Transcript}

One microgram of total RNA was converted into cDNA using Superscript Reverse Transcriptase according to the manufacturer's recommendations (Life Technologies). The quality of the synthesized cDNA was verified by amplification of a 350 bp fragment of the GAPD gene (primers GAPD247f and GAPD596r). One microliter of the $\mathrm{RT}$ reaction $(20 \mu \mathrm{l})$ was then used as template for real time detection of the API2-MLT fusion transcript using the TaqMan Universal PCR master mix and an ABI Prism 7700 sequence detector (Perkin-Elmer Corp.). For the detection of breakpoints upstream of bp 814 of $M L T$, primer set D was used: $15 \mathrm{pmol} M L T-876 \mathrm{r}$ and $20 \mathrm{pmol}$ Taqman probe $M L T-845 \mathrm{r}$ were used in combination with $15 \mathrm{pmol}$ of $A P / 2$ primer $\mathrm{A} 7 \mathrm{f} 1$ in a $50-\mu \mathrm{l}$ reaction. For the detection of breakpoints upstream of bp 1150 of $M L T$, set E was used: $15 \mathrm{pmol} M L T-1233 \mathrm{r}$ and 20 pmol Taqman probe $M L T-1197 \mathrm{r}$ were used in combination with 15 pmol A7f1. Amplification conditions are 2 minutes at $94^{\circ} \mathrm{C}$, followed by 40 cycles of denaturation $\left(94^{\circ} \mathrm{C}, 20\right.$ seconds) and extension $\left(60^{\circ} \mathrm{C}, 1\right.$ minute).

\section{Results}

\section{Histological Findings}

All lymphomas were of peripheral B cell type and could be subdivided into four groups (Table 2). The first group of 23 cases was diagnosed as marginal zone cell lymphoma (MZCL) of extranodal MALT type. Three of these cases were also documented by frozen blocks from in- volved mesenteric lymph nodes. These biopsies showed a lymphomatous process with similar features to the neoplasm found in the gastrectomy specimen. In most cases reactive follicle centers surrounded by a preserved lymphocytic corona were easily found entrapped within the neoplastic marginal zone cell proliferation. The latter was composed of a rather monomorphic, small to medium sized population displaying features of marginal zone cells (centrocyte-like cells) admixed with very few, isolated large cells.

In the 14 cases included in the second group, a similar MALT type lymphoma was observed but associated with a significant large cell proliferation. The large cells either colonized pre-existing follicle centers (in 7 cases) or occurred in sheets and clusters within the marginal zone cell proliferation (in 3 cases). The remaining 4 cases comprised a tumor mass composed of large neoplastic B cells associated with remnants of MALT type lymphoma; the latter either flanked the tumor mass or was detected in other tissue blocks taken from the same gastrectomy specimen. In two of the latter cases the frozen block used for molecular genetic analysis consisted exclusively of large cells. All 14 specimens were diagnosed as extranodal MALT type lymphoma with a large cell component.

The third group comprised 18 cases in which the lymphomatous process was composed entirely of a large B cell proliferation. No genuine MALT type lymphoma component was found, though an atrophic lymphocytic gastritis could be noted in the surrounding of the lymphoma in most of the cases. All cases included in this group were diagnosed as de novo diffuse large B cell lymphoma (DLCL) of the stomach.

The last group consisted of three cases that showed features of Burkitt's/Burkitt's-like lymphoma with the presence of high mitotic rate and large numbers of apoptotic cells.

\section{Molecular Genetic Analysis}

For the detection of an API2-MLT fusion transcript, nested amplification (primer set A) was performed on cDNA derived from $400 \mathrm{ng}$ of total RNA extracted from the lymphoma samples after quality control of the RNA. For this control experiment a 900-bp GAPD fragment was amplified starting from the same amount of RNA in a single round of amplification (30 cycles). To identify genuine API2-MLT fusion transcripts, Southern blots containing the reaction products were hybridized with internal API2 and MLT oligonucleotide probes. For both genes these probes are located in the same exon as the primers used for nested PCR. 
A.

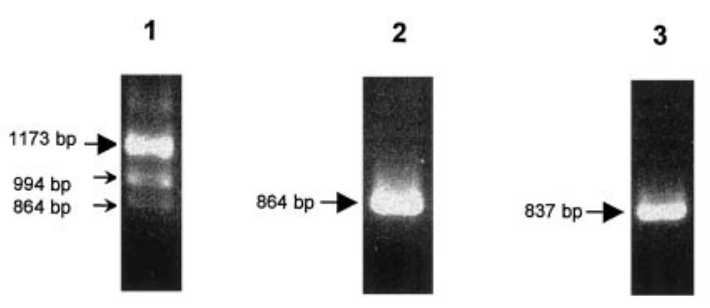

B.

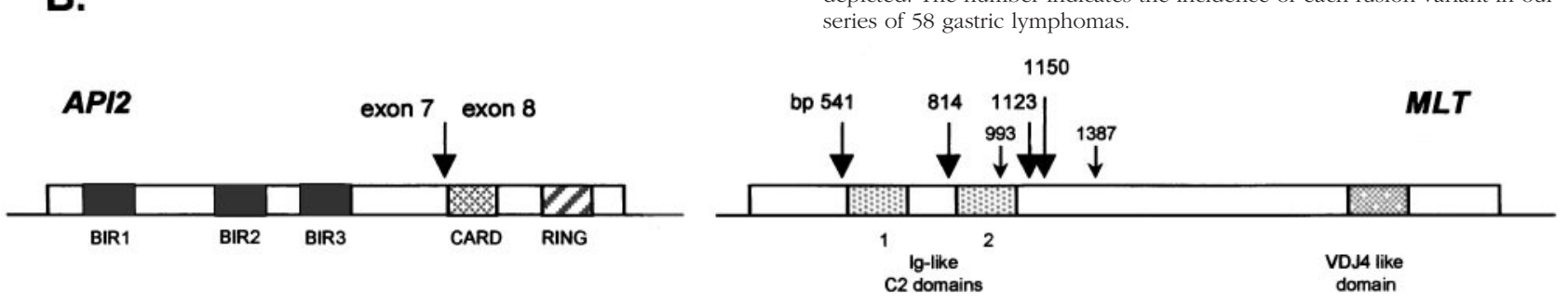

Figure 1. RT-PCR analysis of the API2-MLT fusion transcripts. A: Ethidium bromide staining of the different API2-MLT PCR products obtained after nested amplification with primer set A in case the breakpoint is upstream of MLT basepair 814 (lane 1), 1123 (lane 2), or 1150 (lane 3). The lengths of the amplicons are specified. The smaller arrows (lane 1) indicate the products resulting from alternative splicing. B: The structure of the API2 and $M L T$ CDNA is shown. API2 contains three amino terminal baculovirus IAP repeat domains separated from the carboxyterminal RING domain by a CARD domain. The arrow indicates the position of the API2 breakpoin observed in all cases between exon 7 and exon $8 .{ }^{14}$ MLT harbors two Ig-like C2 domains and an Ig $\gamma$ VDJ4-like sequence. Large arrows depict the position of the different MLT breakpoints, smaller ones the alternative splice sites used when a breakpoint upstream of bp 814 occurs. Numbering is according to $M L T$ accession number AF130356. C: Structure of the different fusion cDNAs. The positions of primer sets A, D, and E (arrows) and probes (small filled rectangles) used in the API2-MLT RT-PCR experiments are depicted. The number indicates the incidence of each fusion variant in our series of 58 gastric lymphomas.

C.

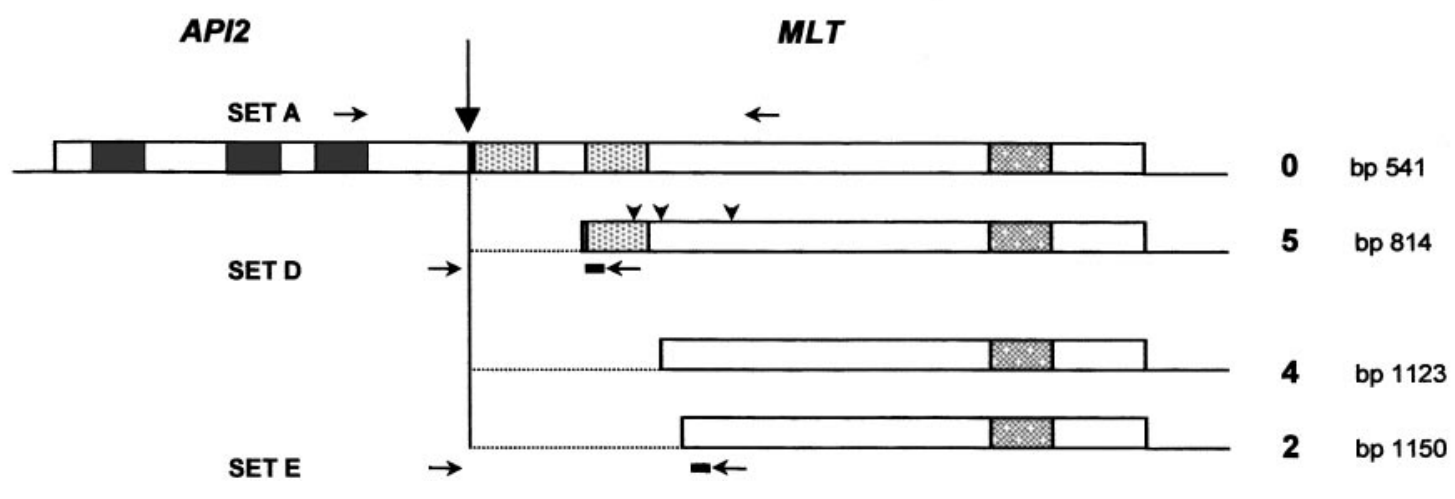

Fragments hybridizing with both the API2 and MLT oligonucleotide probes were detected in biopsies of 11 of 58 patients analyzed (Table 2). In one additional case an aspecific product was amplified as it hybridized exclusively with the API2 probe but not with the MLT oligonucleotide. This was confirmed by sequencing showing the presence of genomic sequences flanking exon 7 of APl2 in the aberrant RT-PCR product.

In almost half of the positive cases (5/11), a PCR fragment of approximately $1.2 \mathrm{~kb}$ was observed accompanied by one or two smaller, less intense bands (Figure 1A). All bands hybridized with the internal oligonucleotide probes for API2 and $M L T$, respectively. Sequence analysis of these different amplification products revealed a fusion of exon 7 of API2 to respectively bp 814, 993, or 1123 of $M L T$ (according to accession number AF130356). A fourth, even shorter transcript was present among the cloned amplification products that fused exon 7 of API2 to bp 1387 of MLT, a fragment not detected by Southern hybridization. All fusions except that to bp 993 of $M L T$ generate in-frame API2-MLT transcripts. The latter induces a switch in reading frame resulting in a TAA stop in the $M L T$ sequence $52 \mathrm{bp}$ beyond the junction.
In the remaining 6 cases, in-frame fusions of exon 7 of API2 to MLT at bp 1123 were detected four times. The other two cases revealed an in-frame fusion to $M L T$ at bp 1150. None of the samples showed a fusion to bp 541 of MLT as we observed previously in one case. ${ }^{11}$ The various transcripts found in the positive cases are shown in Figure 1C. The results on the mesenteric lymph nodes available for two positive cases were identical to the result obtained on the corresponding gastric sample.

Clustering of the breakpoints in intron 7 of API2 indicates either the vulnerability of this region for recombination or merely the portion of the API2 protein required in the fusion protein to exert its function. RT-PCR analysis with primers in exon 7 of $A P I 2$ and a $M L T$ primer at its stop codon (set C) did not identify additional cases, suggesting also an essential contribution of $M L T$ to the fusion protein.

Next we assessed the feasibility of the RT-PCR approach on an $\mathrm{ABI}$ Prism 7700 sequence detector. All cases demonstrating the $A P I 2-M L T$ fusion had their breakpoint in intron 7 of $A P I 2$. The fraction of the MLT gene fused to exon 7 of APl2 varied, however. Therefore, 


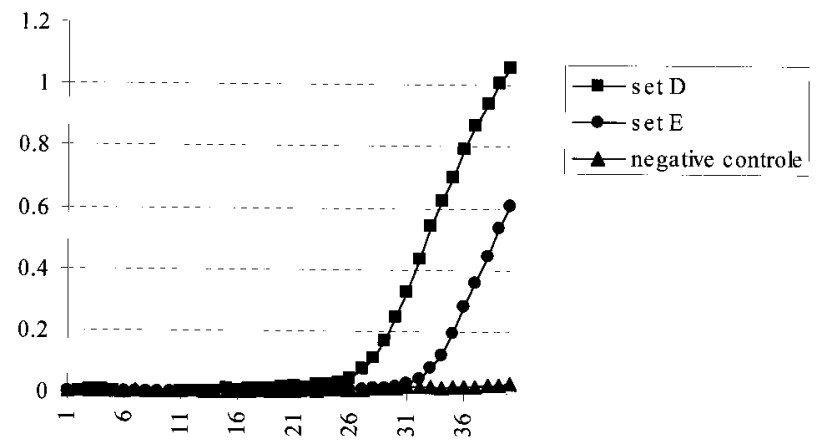

Figure 2. Real time detection of the API2-MLT fusion transcript. The amplification profile of a case with a breakpoint upstream of bp 814 of $M L T$ is shown. The curve with the lowest $C_{T}$ value, 25 , is generated with primer set $\mathrm{D}$, designed for the detection of a breakpoint at this position. The second curve has a higher $\mathrm{C}_{\mathrm{T}}$ value, 32 , and is generated from the same cDNA sample with primer set E. It represents the amplification of a shorter, alternatively spliced API2-MLT fusion transcript that can be detected with this primer set. $x$-axis, cycle number; $y$-axis, $\mathrm{R}_{\mathrm{n}}=$ emission intensity of the reporter dye/emission intensity of the passive reference.

two separate RT-PCR reactions had to be optimized to keep the amplicon length below 300 to $400 \mathrm{bp}$, a prerequisite to retain maximum sensitivity. As the APl2 sequence turned out to be unsuitable, TaqMan probes were derived for the $M L T$ sequence included in the fusion product. Primer set D aimed at the detection of breakpoints upstream of bp 814 of $M L T$, the other (E) upstream of bp 1150 (Materials and Methods, Figure 1C). Firststrand cDNA was synthesized from all 58 RNA samples, and their quality was verified by amplifying a 350-bp fragment of the GAPD gene. Then both API2-MLT reactions were performed on the 58 cDNA samples. As a measure of the accumulation of an amplification product the threshold value $\left(\mathrm{C}_{\mathrm{T}}\right)$ was used. The $\mathrm{C}_{\mathrm{T}}$ value indicates the cycle at which a statistically significant increase in emission intensity of the reporter dye of the probe can be observed in the cDNA sample versus the negative control. Only the 11 cases demonstrating an API2-MLT fusion with the initial RT-PCR approach showed accumulation of a PCR product by real time detection. The six cases with breakpoints upstream of bp 1123/1150 of MLT showed only amplification with primer set $\mathrm{E}$. The other five cases with their breakpoint upstream of bp 814 gave signals with both primer sets. The $\mathrm{C}_{\mathrm{T}}$ values of 23 to 25 obtained with set $D$ were consistently lower than those of 30 to 32 obtained with set E (Figure 2), which could be explained by the larger size of the generated amplicon (601 bp with set E vs. 244 bp with set D). Gel electrophoresis of the amplification products obtained with set E, however, showed a single amplification product of approximately $300 \mathrm{bp}$ (results not shown). This corresponds to the shortest, alternatively spliced product observed in our initial experiments that will have a length of 292 bp with set $E$. Its lower level of expression explains the higher $\mathrm{C}_{\mathrm{T}}$ values observed when compared to set $\mathrm{D}$. The six cases with a breakpoint upstream of bp 1123 or 1150 showed amplification with primer set $\mathrm{E}$, and their $\mathrm{C}_{\mathrm{T}}$ values were around 25.

\section{Correlation of Molecular Results to the Histological Findings}

All 11 positive cases were diagnosed as extranodal MALT type lymphoma without large cell component. These cases did not significantly differ, regarding clinical characteristics and morphological and phenotypic features, from the MALT type lymphomas without large cell component, lacking the translocation. In addition, no correlation was found between the molecular findings and the $H$. pylori status.

\section{Discussion}

Only a limited number of marginal zone cell lymphomas, including MALT type lymphoma, documented by clonal cytogenetic findings have been reported in the literature. Most of these studies stressed the occurrence of trisomy 3 in a high percentage of extranodal, nodal, and splenic cases but failed to detect $t(11 ; 18)$ by classical cytogenetic investigation. ${ }^{8,9}$ More recently the latter translocation has been recognized as a recurrent structural abnormality in extranodal MALT type Iymphoma. ${ }^{10,12,13}$ It was identified in 4 of 10 gastrectomy specimens diagnosed as extranodal low grade MALT type lymphoma, all lacking any large cell component. ${ }^{10}$

In the present study we detected $t(11 ; 18)(q 21 ; q 21)$ by RT-PCR in 11 cases studying a series of 58 consecutive gastrectomy specimens diagnosed as primary nonHodgkin's lymphoma of the stomach. The developed RT-PCR approach aimed at the detection of API2-MLT fusion transcripts beyond exon 3 of the APl2 gene. ${ }^{14}$ This exon was originally chosen because it contains the ATG start codon and two and a half of the three baculovirus IAP repeat domains with the anti-apoptotic properties of the protein. A second round of nested amplification was routinely performed to amplify the signal from those RNA samples of marginal quality, as observed after GAPD control amplification, and/or with a limited portion of tumor mass in the specimen. Southern hybridizations with internal API2 and MLT oligonucleotides proved that all but one of the amplified fragments represented genuine API2-MLT fusions.

The breakpoints on chromosome 18 were scattered throughout the $M L T$ gene. In half of the positive cases (5/11), exon 7 of $A P I 2$ is fused with bp 814 of $M L T$. However one or two smaller, less intense bands could always be observed and sequence analysis revealed an even shorter fourth one. Sequence analysis of genomic clones demonstrated $3^{\prime}$ splice consensus sequences flanking the $M L T$ sequence at positions 814, 993, 1123, and 1387, respectively (Baens M, Steyls A, Dierlamm J, De Wolf-Peeters C, Marynen P, manuscript submitted). Therefore, the genomic breakpoints in these cases are most likely located in the intron preceding an $M L T$ exon starting at bp 814 and alternative splicing generates variable API2-MLT transcripts. Fusions between exon 7 of API2 and bp 1123 or bp 1150 of $M L T$ were observed four and two times respectively, whereas an API2-MLT fusion identical to that of case 2 from our previous report ${ }^{11}$ was 
not detected in this study. For all fusions it was confirmed that the MLT sequences attached to exon 7 of API2 represent proper exons (Baens et al., submitted).

Clustering of the API2 breakpoints permitted us to set up a RT-PCR approach on an ABI Prism 7700 sequence detector. Two different reactions were optimized that allow identification of fusions between exon 7 of API2 and bp $541 / 814$ or bp $1123 / 1150$ of MLT respectively. All cases diagnosed with an API2-MLT fusion by the original RT-PCR approach were also positive after 40 rounds of real time detection. The increased sensitivity of the real time amplification detection technique allowed us to skip the second round of nested amplification.

The API2-MLT fusion transcript, detected in 11 gastrectomy specimens by conventional RT-PCR and by real time PCR, occurred only in cases diagnosed as MALT type lymphoma, included in group I. None of these cases harbored a significant large cell proliferation defined as follicular colonization, sheets of blast cells or a large cell tumor mass. The incidence of the $t(11 ; 18)$ in low grade MALT type lymphoma reaches $48 \%$ in our study, an incidence that supports a close association of this chromosomal anomaly with this particular subtype of marginal zone cell lymphoma and therefore suggests a role for the API2-MLT transcript in the histogenesis of this lymphoma.

Like Ott et al, ${ }^{10}$ we did not find any $t(11 ; 18)$ positive samples among DLCL, the Burkitt's/Burkitt's-like lymphomas and MALT type lymphomas comprising a large cell proliferation, so quoted high grade component. MZCL including low grade MALT lymphomas are composed predominantly of small to medium sized cells responsible for the characteristic picture at low power and only a few larger, stimulated cells can be found admixed. The mechanisms involved in the occurrence of a clear-cut large cell component within this lymphoma, generally indicated as high grade MALT Iymphoma, remain enigmatic. Data provided by immunohistochemical stainings and molecular analysis support investigators considering the presence of a large cell component within MALT type lymphoma as a transformation and/or progression from a low grade lymphoma into a high grade disease. Both techniques revealed the same light chain restriction and/or the same $\mathrm{IgH}$ rearrangements in the two components of the lymphomatous process as performed in a limited number of cases. ${ }^{15}$ Based on these results it was even postulated that the vast majority, if not all, lymphomas of the stomach should be considered as extranodal MALT type lymphomas. From that point of view gastric neoplasms composed only of large cells merely represent transformed small cell malignancies of which the pre-existing lymphoma is no longer detectable due to overgrowth by the transformed population. ${ }^{5}$ The exclusive occurrence of $\mathrm{t}(11 ; 18)$ by RT-PCR in low grade MALT type lymphoma points toward the distinct character of the extranodal MZCL of MALT type occurring in the gut. As far a large cell component occurring in this lymphoma is indeed related to the MALT type lymphoma ${ }^{15,16}$ or merely represents a composite lymphoma comprising a diffuse large B cell lymphoma and a MALT type lymphoma as suggested by others ${ }^{17}$ is still problematic. Nevertheless, our data fully support a clear distinction of
MALT type lymphoma from diffuse large B cell lymphoma of the stomach.

We previously demonstrated that the $t(11 ; 18)$ was accompanied by a cryptic deletion of the $3^{\prime}$ part of the API2 gene, which precludes the expression of the reciprocal $M L T-A P I 2$ transcript and attributes the oncogenic properties to the API2-MLT fusion protein. ${ }^{11}$ RT-PCR analysis of the reciprocal MLT-API2 transcript demonstrated expression in no more than 3 cases documented with the $t(11 ; 18)$, including case 2 from our previous study. ${ }^{11}$ This observation further strengthens the importance of the API2-MLT fusion protein, although we cannot exclude that in rare cases variant breakpoints might occur, eg, within the 3' UTR of MLT. Recent studies showed that a construct containing only the baculovirus IAP repeat domains of API2 is sufficient for inhibition of caspases and suppression of apoptosis. ${ }^{18}$ However, the present study supports a specific role for the MLT portion in the fusion protein as in all cases either an in-frame fusion with $M L T$ is created, or alternative splicing occurs generating both in-frame and out-of-frame products. In the latter case the functional in-frame fusion products are clearly the more abundant.

In conclusion, we present a RT-PCR technique to detect $\mathrm{t}(11 ; 18)$ in a minimal amount of RNA material obtained from frozen tissue sections. The translocation was found in almost half of the cases diagnosed as MALT type lymphoma on a gastrectomy specimen. Consequently, application of this technique on endoscopic biopsies will provide a new and very instructive additional diagnostic tool to distinguish between reactive lymphoid infiltrates and MALT type lymphoma in patients affected by H. pylori infection.

\section{Acknowledgments}

We thank Miet Vanherck for expert technical assistance.

\section{References}

1. Harris NL, Jaffe ES, Stein H, Banks PM, Chan JK, Cleary ML, Delsol G, De-Wolf-Peeters C, Falini B, Gatter KC: A revised EuropeanAmerican classification of lymphoid neoplasms: a proposal from the International Lymphoma Study Group [see comments]. Blood 1994, 84:1361-1392

2. Isaacson PG, Spencer J: Malignant lymphoma of mucosa-associated lymphoid tissue. Histopathology 1987, 11:445-462

3. Wotherspoon AC, Ortiz HC, Falzon MR, Isaacson PG: Helicobacter pylori-associated gastritis and primary B-cell gastric lymphoma [see comments]. Lancet 1991, 338:1175-1176

4. Chan JK, Ng CS, Isaacson PG: Relationship between high-grade lymphoma and low-grade B-cell mucosa-associated lymphoid tissue lymphoma (MALToma) of the stomach. Am J Pathol 1990, 136:11531164

5. Isaacson PG: Recent developments in our understanding of gastric lymphomas. Am J Surg Pathol 1996, 20(suppl. 1):S1-S7

6. De Wolf-Peeters C, Achten R: The histogenesis of large-cell gastric lymphomas [comment]. Histopathology 1999, 34:71-75

7. De Wolf-Peeters C, Tierens A. Controversies in MALT Iymphoma classification, low and high grade. Histopathology 1998, 32:277-278

8. Wotherspoon AC, Pan LX, Diss TC, Isaacson PG: Cytogenetic study of B-cell lymphoma of mucosa-associated lymphoid tissue. Cancer Genet Cytogenet 1992, 58:35-38 
9. Dierlamm J, Pittaluga S, Wlodarska I, Stul M, Thomas J, Boogaerts M, Michaux L, Driessen A, Mecucci C, Cassiman J-J, De Wolf-Peeters C, Van Den Berghe H: Marginal zone B-cell lymphomas of different sites share similar cytogenetic and morphologic features. Blood 1996, 87:299-307

10. Ott G, Katzenberger T, Greiner A, Kalla J, Rosenwald A, Heinrich U, Ott MM, Muller-Hermelink HK: The t(11;18)(q21;q21) chromosome translocation is a frequent and specific aberration in low-grade but not high-grade malignant non-Hodgkin's lymphomas of the mucosaassociated lymphoid tissue (MALT-) type. Cancer Res 1997, 57: 3944-3948

11. Dierlamm J, Baens M, Wlodarska I, Stefanova-Ouzounova M, Hernandez JM, Hossfeld DK, De Wolf-Peeters C, Hagemeijer A, Van Den Berghe $\mathrm{H}$, Marynen P: The apoptosis inhibitor gene APl2 and a novel $18 \mathrm{q}$ gene, MLT, are recurrently rearranged in the $\mathrm{t}(11 ; 18)(\mathrm{q} 21 ; \mathrm{q} 21)$ associated with MALT lymphomas. Blood 1999, 93:3601-3609

12. Auer IA, Gascoyne RD, Connors JM, Cotter FE, Greiner TC, Sanger WG, Horsman DE: $\mathrm{t}(11 ; 18)(\mathrm{q} 21 ; \mathrm{q} 21)$ is the most common translocation in MALT Iymphomas. Ann Oncol 1997, 8:979-985

13. Horsman D, Gascoyne R, Klasa R, Coupland R: t(11;18)(q21;q21:1): a recurring translocation in lymphomas of mucosa-associated lymphoid tissue (MALT)? Genes Chromosomes Cancer 1992, 4:183-187

14. Young SS, Liston P, Xuan JY, McRoberts C, Lefebvre CA, Korneluk RG: Genomic organization and physical map of the human inhibitors of apoptosis: HIAP1 and HIAP2. Mamm Genome 1999, 10:44-48

15. Peng H, Du M, Diss TC, Isaacson PG, Pan L: Genetic evidence for a clonal link between low and high-grade components in gastric MALT B-cell lymphoma. Histopathology 1997, 30:425-429

16. Montalban C, Manzanal A, Castrillo JM, Escribano L, Bellas C: Low grade gastric B-cell MALT lymphoma progressing into high grade Iymphoma: clonal identity of the two stages of the tumour, unusual bone involvement and leukemic dissemination. Histopathology 1995, 27:89-91

17. Matolcsy A, Nagy M, Kisfaludy N, Kelenyi G: Distinct clonal origin of low-grade MALT-type and high-grade lesions of a multifocal gastric lymphoma [see comments]. Histopathology 1999, 34:6-8

18. Roy N, Deveraux QL, Takahashi R, Salvesen GS, Reed JC: The C-IAP-1 and C-IAP-2 proteins are direct inhibitors of specific caspases. EMBO J 1997, 16:6914-6925 